\title{
The Feasibility of COPD Surveillance in Ontario: A Population Study
}

\author{
J. Charles Victor, Teresa To, Andrew Wilton, Jun Guan, Minnie H. Ho and Andrea S. Gershon
}

\section{The Issue}

Chronic obstructive pulmonary disease (COPD), also known as emphysema or chronic bronchitis, is a progressive chronic respiratory disorder characterized by non-reversible obstruction of the airways in the lungs. It is characterized clinically by shortness of breath, cough, sputum production, wheeze and limited exercise tolerance and can lead to respiratory failure and death. While prevalence estimates vary widely depending on geographical and socio-demographic patterns, the prevalence of COPD remains high in adults over the age of 40 years, ranging from 2 to $22 \%$ depending on the definition used and the method for ascertaining positive COPD status (Gershon et al. 2010; Halbert et al. 2006). Despite the high prevalence in the general population, several authors note that COPD remains under-diagnosed globally (Hill et al. 2010; Huchon et al. 2002; Schirnhofer et al. 2007; Zielinski et al. 2006). This under-diagnosis has often been attributed to a lack of symptom recognition in patients who do not have obvious risk factors (Buist et al. 2007); however, others have confirmed that the problem exists in those with obvious risk factors, such as a history of smoking (Hill et al. 2010).

Mild to severe forms of COPD are associated with considerable mortality, morbidity and decreased quality of life (Garrido et al. 2006). COPD is currently the fourth leading cause of death both in Canada and globally (Buist et al. 2007; O'Donnell et al. 2007), and among chronic diseases it is the only leading cause of death that is increasing (Lopez et al. 2006). It is projected that COPD will be the fifth leading cause of disability-adjusted life years (DALYs) globally by 2020 (Murray and Lopez 1997).

Despite its substantial impact, COPD does not receive the same recognition as other diseases of comparable burden (Barnes and Kleinert 2004). It has been suggested that in developed nations such a lack of public awareness of the disease and its co-morbidities is in part due to the view that COPD is a consequence of people's own actions (i.e., smoking) (Barnes and Kleinert 2004). For example, a recent analysis by the UK Clinical Research Collaboration (2006) found that respiratory diseases (including COPD) rated fourth highest in terms of DALYs but only 13 th highest in terms of research spending - the largest discrepancy between disease burden and spending of all the disease areas studied. Similar findings have been demonstrated for COPD funding in the United States (Gillum et al. 2011).

Given the immense burden that COPD places on the individual and society in terms of mortality, morbidity and direct and indirect costs, several organizations (Centers for Disease Control and Prevention 2011; European Respiratory Society 2011; Public Health Agency of Canada 2011) have called for COPD surveillance systems to aid in the development and targeting of public health programs and policies. There are several challenges to developing such a comprehensive population-based surveillance system (Chapman et al. 2006). First, one of the most important barriers has been the lack of a clear definition of COPD (Chapman et al. 2006; Mannino 2002). Second, population-based surveillance systems by nature are costly both in time and resources. And, third, it has been suggested that public support of healthcare funding for the surveillance of a disease perceived to affect mostly the elderly (Chapman et al. 2006) and smokers (Voelkel 2000) might be weak. A solution to some of these challenges might be the use of health administrative data as a surveillance tool. In Ontario, we have access to universal healthcare administrative databases containing information on hospitalizations, ambulatory visits and emergency visits linked together at the individual level. Given the capacity to follow a large and complete population, this study was undertaken to (1) determine the feasibility of using health administrative databases for the surveillance of COPD; and (2) provide an indication of the prevalence, incidence and mortality associated with COPD in the Ontario population. Furthermore, regional-level analysis was completed to demonstrate how variation in COPD rates might be used to identify areas with high current and future burden and to assist in policy and program implementation.

\section{The Study}

Healthcare administrative databases and a validated case definition for identifying physician-diagnosed COPD were used to identify Ontarians from 35 to 99 years of age who had COPD between 1996-1997 and 2009-2010. In brief, individuals aged 35 years and older were considered to have 
COPD if they had at least one Canadian Institute for Health Information Discharge Abstract Database (CIHI-DAD) hospitalization record or one Ontario Health Insurance Plan (OHIP) physician billing for chronic bronchitis, COPD or emphysema (Gershon et al. 2009). The sensitivity of this case definition is $85.0 \%$, and the specificity is $78.4 \%$ (Gershon et al. 2009). Annual age- and sex-standardized prevalences, incidences and all-cause mortalities (among Ontarians with COPD) were determined. Furthermore, age- and sex-standardized prevalences, incidences and all-cause mortalities for fiscal year 2009-2010 were calculated stratified by Local Health Integration Networks (LHINs - the 14 organizational bodies responsible for planning funding to local healthcare services in Ontario) to determine the degree of regional variation for each of these indices. All analyses were conducted in SAS v. 9.2 (Cary, North Carolina), and all rates were standardized to the Ontario component of the 2001 Census of Canada.

\section{Key Findings}

\section{Prevalence of COPD}

Overall age- and sex-standardized prevalence rates increased from 7.7 per 100 persons 35 years and older in 1996-1997 to 9.9 per 100 in 2009-2010; however, most of this increase was seen in the earlier years, and the prevalence rate increased only marginally from 2006-2007 onward. Females experienced a greater increase over this period (from 6.8 to 9.4 per 100) compared with males (from 9.0 to 10.6 per 100).

In 2009-2010, geographical variation was substantial, with four of the 14 LHINs having age- and sex-standardized prevalence rates more than $20 \%$ above the overall Ontario rate of 9.9 per 100, and three LHINs having prevalence rates more than $20 \%$ below the overall Ontario rate (Figure 1).

\section{Incidence of COPD}

Incidence rates, in contrast, have been declining over the past 14 years, with the overall incidence being 12.5 per 1,000 in 1996-1997 and 9.1 per 1,000 in 2009-2010. In keeping with the observed trends in prevalence, rates declined less for females, who had a $24 \%$ relative reduction, than for males, who experienced a $31 \%$ relative reduction.
In 2009-2010, the geographical variation in incidence mirrored that of the prevalence rates, with a few key exceptions (Figure 2). While the prevalence rate for Southwestern Ontario was similar to the overall provincial rate (9.9 per 100), the incidence rate for Southwestern Ontario was approximately $15 \%$ (relative) higher than the Ontario rate. Of notable concern are the Erie St. Clair, South East and North East LHINs, all of which exceeded overall Ontario prevalence and incidence rates by more than $20 \%$.

\section{All-Cause Mortality of Ontarians with COPD}

Age- and sex-adjusted all-cause mortality rates among individuals with physician-diagnosed COPD decreased from 5.6 per 100 to 3.9 per 100 over the 14 -year period. Most of these reductions were seen in males, who experienced a $37 \%$ relative decrease in mortality rate (versus $23 \%$ for females with COPD) during this period. 
FIGURE 2.

Age- and sex-adjusted incidence of COPD per 1,000 population aged 35 years and older in Ontario, by LHIN, 2009-2010

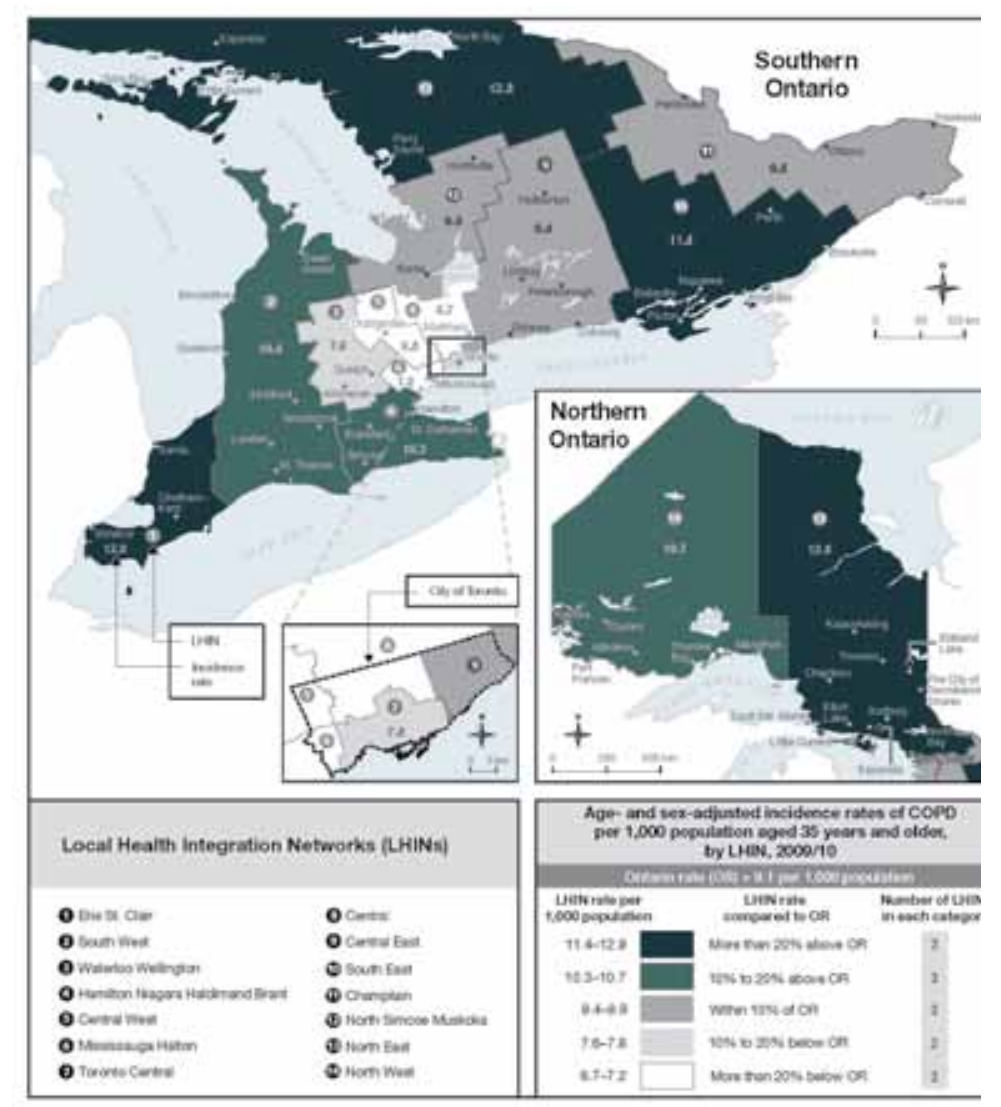

COPD = chronic obstructive pulmonary disease; $\mathrm{LHIN}=$ local health integration network a slower rate since 2006-2007. Although age- and sex-adjusted incidence rates are declining, the overall annual incidence rate remains close to $1 \%$. All-cause mortality rates are declining, resulting in part in the increase in prevalence. These findings suggest that the burden that COPD places on individuals and the healthcare system will continue to grow for several decades. These findings confirm other estimates of present and future burdens in Canada and other developed nations based on smaller population-based samples and selfreported survey data (Chapman et al. 2003; Dal Negro et al. 2002; Fukuchi et al. 2004; Nielsen et al. 2009).

Our work expands on previous findings by being able to examine COPD at the regional level. Because the Ontario healthcare administrative databases capture healthcare utilization for almost the complete population, estimates of prevalence and incidence can be made at smaller regional levels and with almost universal representativeness. This study found that within Ontario, COPD prevalence and incidence vary widely by LHINs. Both prevalence and incidence were found to range between $20 \%$ higher and $20 \%$ lower relative to the overall Ontario rates, suggesting that some regions currently experience a considerably higher burden and costs due
There was very little geographical variation in 2009-2010 mortality rates across the province (Figure 3 ), with 12 of the 14 LHINs having standardized rates within $10 \%$ of the overall Ontario rate (3.9 per 100). Only the Central LHIN (3.3 per $100)$ and the Waterloo Wellington LHIN (4.3 per 100) differed by greater than $10 \%$ (relative).

\section{Discussion}

This study demonstrates that the universal healthcare administrative databases available in Ontario (containing information on hospitalizations, ambulatory visits and emergency visits) can be linked together and to demographic information from Statistics Canada to create a detailed and highly representative surveillance system that may be used to estimate the prevalence, incidence, all-cause mortality and regional variation associated with physician-diagnosed COPD. Of particular concern is the confirmation that prevalence rates continue to increase, albeit at to COPD and will continue to do so into the future. This may have considerable implications for policies and, in particular, the development and management of programs related to COPD management, including those related to long-term care and home care. Furthermore, the ability to provide COPD surveillance at the regional level allows for targeted COPD-prevention campaigns.

\section{The ability to provide COPD surveillance at the regional level allows for targeted COPD-prevention campaigns.}

This study has some limitations. First, the classification of individuals with COPD based on healthcare claims may result in some misclassification. However, the case definition used to identify individuals with COPD has been validated against 
patient charts and found to have good sensitivity and specificity. Furthermore, this case definition identifies actual healthcare claims for COPD (i.e., payments made by the province of Ontario for services related to COPD), regardless of the actual status of the patient. Therefore, the prevalence and incidence derived from this case definition can be viewed as representing only physician-diagnosed COPD. Given that physician-diagnosed COPD has been shown to be an underestimate of true COPD in the population (Hill et al. 2010), this case definition may therefore reflect an underestimate of the true prevalence and incidence (Gershon et al. 2009). This, in turn, suggests that the current and future burdens of COPD suggested by these data are conservative.

In conclusion, healthcare administrative databases provide a powerful and costeffective tool for the surveillance of COPD and the estimation of its present and future burdens. In Ontario, the prevalence and incidence of COPD vary widely across regions. Several regions demonstrate much higher COPD prevalences and incidences, suggesting that they will have considerable future burden. Policies and programs aimed at COPD prevention and management are needed to target high-risk regions that demonstrate considerable burden.

\section{Acknowledgements}

Funding for this project was made available from the Canadian Institutes of Health Research, the Public Health Agency of Canada and the Ontario Ministry of Health and Long-Term Care. The project was also supported by the Institute for Clinical Evaluative Sciences (ICES), which is funded in part by an annual grant from the Ontario Ministry of Health and Long-Term Care. Dr. Gershon is supported by a Career Scientist Award from the Ontario Ministry of Health and Long-Term Care. Dr. To is supported by the Dales Award in Medical Research from the University of Toronto. The opinions, results and conclusions reported in this article are those of the authors and are independent of the funding sources. HQ

\section{References}

Barnes, P.J. and S. Kleinert. 2004. "COPD - A Neglected Disease.” Lancet 364(9434): 564-65.

\section{FIGURE 3. \\ Age- and sex-adjusted mortality rates per 100,000 population 2009-2010}

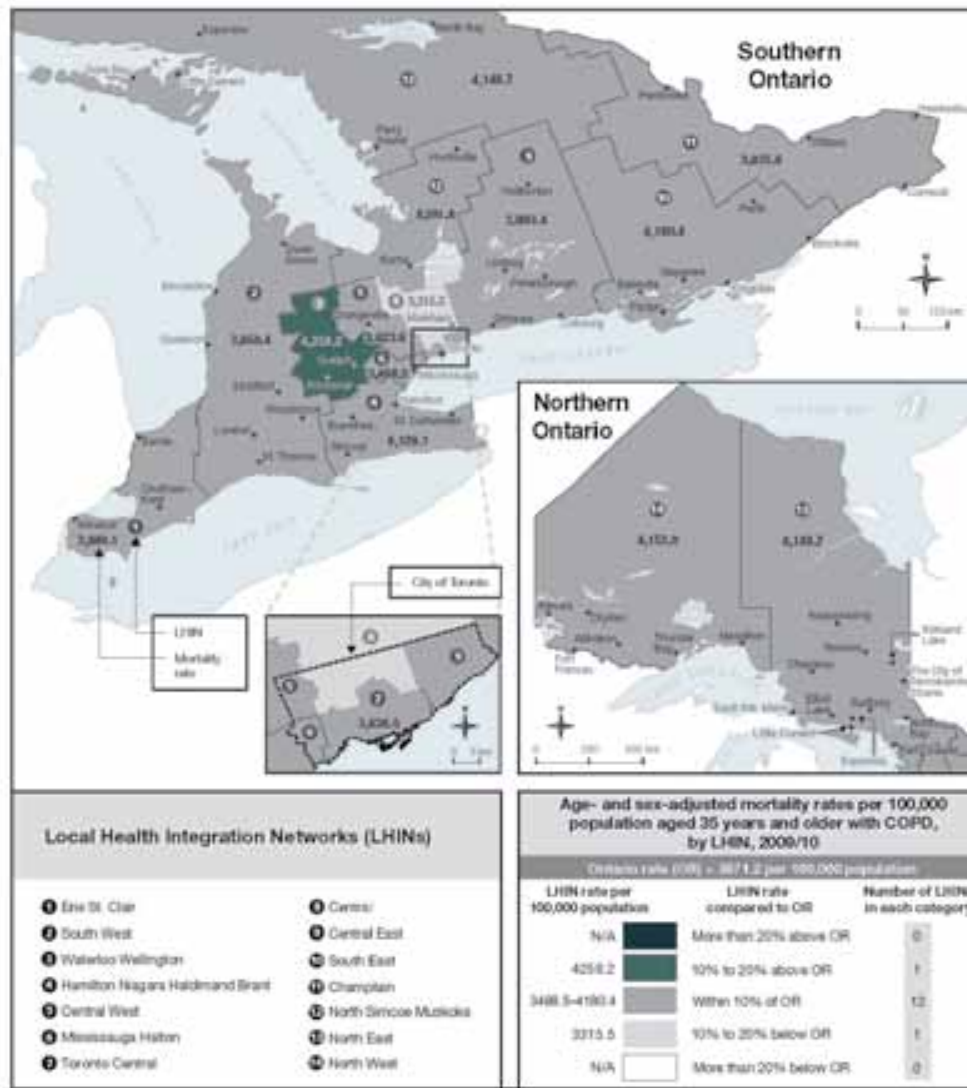

COPD = chronic obstructive pulmonary disease; $\mathrm{LHIN}=$ local health integration network.

Buist, A.S., M.A. McBurnie, W.M. Vollmer, S. Gillespie, P. Burney, D.M. Mannino et al. 2007. "International Variation in the Prevalence of COPD (the BOLD Study): A Population-Based Prevalence Study." Lancet 370(9589): 741-50.

Centers for Disease Control and Prevention. 2011. Public Health Strategic Framework for COPD Prevention. Atlanta, GA: Author. Retrieved July 13, 2011. <http://www.cdc.gov/copd/pdfs/Framework_ for_COPD_Prevention.pdf $>$.

Chapman, K.R., D.M. Mannino, J.B. Soriano, P.A. Vermeire, A.S. Buist, M.J. Thun et al. 2006. "Epidemiology and Costs of Chronic Obstructive Pulmonary Disease." European Respiratory Journal 27(1): 188-207.

Chapman, K.R., J. Bourbeau and L. Rance. 2003. "The Burden of COPD in Canada: Results from the Confronting COPD Survey." Respiratory Medicine 97(Suppl. C): S23-31.

Dal Negro, R., P. Berto, S. Tognella, L. Quareni and Global Outcomes in Lung Disease Study Group. 2002. "Cost-of-Illness of Lung Disease 
in the TriVeneto Region, Italy: The GOLD Study.” Monaldi Archives for Chest Disease 57(1): 3-9.

European Respiratory Society. 2011. ERS President Calls for EuropeWide COPD Surveillance System. Lausanne, Switzerland: Author. Retrieved July 13, 2011. <http://www.ersnet.org/index.php/news/ item/4045-ers-president-calls-for-europe-wide-copd-surveillancesystem.html>.

Fukuchi, Y., M. Nishimura, M. Ichinose, M. Adachi, A. Nagai, T. Kuriyama et al. 2004. "COPD in Japan: The Nippon COPD Epidemiology Study.” Respirology 9(4): 458-65.

Garrido, P.C., J.M. Diez, J.R. Gutiérrez, A.M. Centeno, E.G. Vázquez, A.G. de Miguel et al. 2006. "Negative Impact of Chronic Obstructive Pulmonary Disease on the Health-Related Quality of Life of Patients. Results of the EPIDEPOC Study." Health and Quality of Life Outcomes 4: 31.

Gershon, A.S., C. Wang, J. Guan, J. Vasilevska-Ristovska, L. Cicutto and T. To. 2009. "Identifying Individuals with Physician Diagnosed COPD in Health Administrative Databases." COPD 6(5): 388-94.

Gershon, A.S., C. Wang, A.S. Wilton, R. Raut and T. To. 2010. “Trends in Chronic Obstructive Pulmonary Disease Prevalence, Incidence, and Mortality in Ontario, Canada, 1996 to 2007: A Population-Based Study." Archives of Internal Medicine 170(6): 560-65.

Gillum, L.A., C. Gouveia, E.R. Dorsey, M. Pletcher, C.D. Mathers, C.E. McCulloch et al. 2011. "NIH Disease Funding Levels and Burden of Disease.” PLoS One 6(2): e16837.

Halbert, R.J., J.L. Natoli, A. Gano, E. Badamgarav, A.S. Buist and D.M. Mannino. 2006. "Global Burden of COPD: Systematic Review and Meta-Analysis." European Respiratory Journal 28(3): 523-32.

Hill, K., R.S. Goldstein, G.H. Guyatt, M. Blouin, W.C. Tan, L.L. Davis et al. 2010. "Prevalence and Underdiagnosis of Chronic Obstructive Pulmonary Disease among Patients at Risk in Primary Care." Canadian Medical Association Journal 182(7): 673-78.

Huchon, G.J., A. Vergnenegre, F. Neukirch, G. Brami, N. Roche and P.M. Preux. 2002. "Chronic Bronchitis among French Adults: High Prevalence and Underdiagnosis." European Respiratory Journal 20(4): 806-12.

Lopez, A.D., K. Shibuya, C. Rao, C.D. Mathers, A.L. Hansell, L.S. Held et al. 2006. "Chronic Obstructive Pulmonary Disease: Current Burden and Future Projections." European Respiratory Journal 27(2): 397-412.

Mannino, D.M. 2002. “COPD: Epidemiology, Prevalence, Morbidity and Mortality, and Disease Heterogeneity." Chest 121(5 Suppl.): 121S-26S.

Murray, C.J.L. and A.D. Lopez. 1997. "Alternative Projections of Mortality and Disability by Cause 1990-2020: Global Burden of Disease Study." Lancet 349(9064): 1498-504.

Nielsen, R., A. Johannessen, B. Benediktsdottir, T. Gislason, A.S. Buist, A. Gulsvik et al. 2009. "Present and Future Costs of COPD in Iceland and Norway: Results from the BOLD Study." European Respiratory Journal 34(4): 850-57.

O’Donnell, D.E., S. Aaron, J. Bourbeau, P. Hernandez, D.D. Marciniuk, M. Balter et al. 2007. "Canadian Thoracic Society Recommendations for Management of Chronic Obstructive Pulmonary Disease - 2007 Update." Canadian Respiratory Journal 14(Suppl. B): 5B-32B.

Public Health Agency of Canada. Chronic Obstructive Pulmonary Disease (COPD). Ottawa, ON: Author. Retrieved July 13, 2011. $<$ http://www.phac-aspc.gc.ca/cd-mc/crd-mrc/copd-mpoc-eng.php>.

Schirnhofer, L., B. Lamprecht, W.M. Vollmer, M.J. Allison, M. Studnicka, R.L. Jensen et al. 2007 "COPD Prevalence in Salzburg, Austria: Results from the Burden of Obstructive Lung Disease (BOLD) Study." Chest 131(1): 29-36.

UK Clinical Research Collaboration. 2006. UK Health Research Analysis. London: Author. Retrieved July 13, 2011. <http://www.ukcrc. org/researchcoordination/healthresearchanalysis/ukanalysis/>.

Voelkel, N.F. 2000. "Raising Awareness of COPD in Primary Care." Chest 117(5 Suppl. 2): 372S-75S.

Zielinski, J., M. Bednarek, D. Górecka, G. Viegi, S.S. Hurd, Y. Fukuchi et al. 2006. "Increasing COPD Awareness." European Respiratory Journal 27(4): 833-52.

\section{About the Authors}

J. Charles Victor, MSc, PStat, is an epidemiologist and research coordination lead for the Chronic Disease and Pharmacotherapy Program at the Institute for Clinical Evaluative Sciences (ICES) in Toronto, Ontario. He is also affiliated with the University of Toronto. He can be contacted by e-mail at charles.victor@ices.on.ca.

Teresa To, PhD, is a senior scientist at The Hospital for Sick Children in Toronto, Ontario, and an adjunct scientist at ICES. She is also a professor at the University of Toronto in the departments of Health Policy, Management and Evaluation; and Pediatrics; the Dalla Lana School of Public Health; and the Institute for Medical Sciences.

Andrew Wilton, MSc, is an analyst at ICES.

Jun Guan, MSc, is a biostatistician and analyst lead for the Health System Planning and Evaluation Program at ICES.

Minnie M. Ho, MHSc, is an epidemiologist and research coordination lead for the Health System Planning and Evaluation Program at ICES.

Andrea S. Gershon, MD, MSc, FRCPC, is a respirologist at Sunnybrook Health Sciences Centre, in Toronto, Ontario, and an adjunct scientist at ICES. She is also affiliated with the University of Toronto and The Hospital for Sick Children.

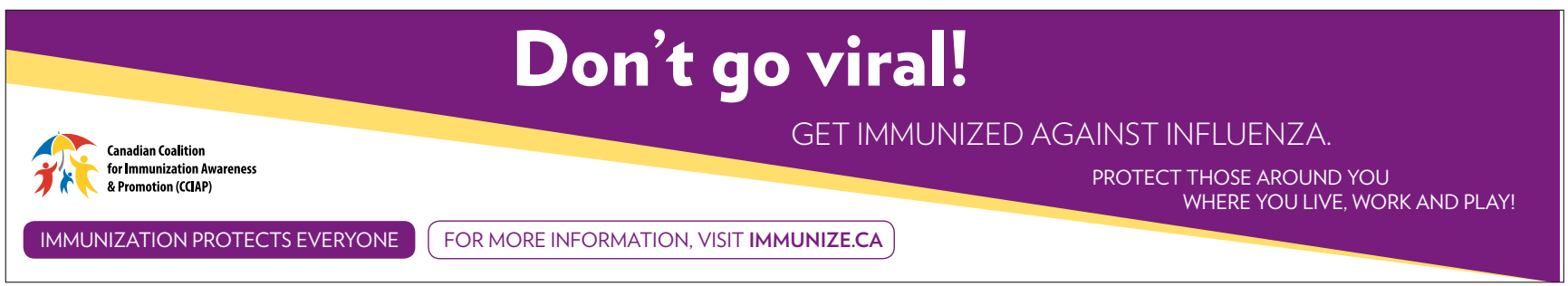

